Published in final edited form as:

AIDS Behav. 2018 February ; 22(2): 479-496. doi:10.1007/s10461-017-1796-4.

\title{
Evidence-Based Identification of Key Beliefs Explaining Infant Male Circumcision Motivation among Expectant Parents in Zimbabwe: Targets for Behavior Change Messaging
}

\author{
Daniel E. Montaño ${ }^{1,2,4}$, Mufuta Tshimanga ${ }^{3}$, Deven T. Hamilton ${ }^{4}$, Gerald Gorn ${ }^{5}$, and Danuta \\ Kasprzyk ${ }^{1,2,4}$ \\ ${ }^{1}$ Department of Family and Child Nursing, University of Washington, Seattle, WA \\ ${ }^{2}$ Department of Global Health, University of Washington, Seattle, WA \\ ${ }^{3}$ Department of Community Medicine, University of Zimbabwe, Harare, Zimbabwe \\ ${ }^{4}$ Center for Studies in Demography and Ecology, University of Washington, Seattle, WA \\ ${ }^{5}$ Department of Management and Marketing, The Hong Kong Polytechnic University, Hong Kong
}

\section{Abstract}

Slow adult male circumcision (VMMC) uptake is one factor leading some to recommend increased priority for infant male circumcision (IMC) in sub-Saharan African countries. This research, guided by the Integrated Behavioral Model (IBM), was carried out to identify key beliefs that best explain Zimbabwean parents' motivation to have their infant sons circumcised. A quantitative survey, designed from qualitative elicitation study results, was administered to independent representative samples of 800 expectant mothers and 795 expectant fathers in two urban and two rural areas in Zimbabwe. Multiple regression analyses found IMC motivation among fathers was explained by instrumental attitude, descriptive norm and self-efficacy; while motivation among mothers was explained by instrumental attitude, injunctive norm, descriptive norm, self-efficacy, and perceived control. Regression analyses of beliefs underlying IBM constructs found some overlap but many differences in key beliefs explaining IMC motivation among mothers and fathers. We found differences in key beliefs among urban and rural parents. Urban fathers' IMC motivation was explained best by behavioral beliefs, while rural fathers' motivation was explained by both behavioral and efficacy beliefs. Urban mothers' IMC motivation was explained primarily by behavioral and normative beliefs, while rural mothers' motivation was explained mostly by behavioral beliefs. The key beliefs we identified should serve as targets for

Corresponding Author: Daniel E. Montaño, Department of Family and Child Nursing, School of Nursing, Box 357262, University of Washington, Seattle, WA 98195-7920, Phone: 206-524-9314; Fax: 206-543-9266, montano@uw.edu.

Compliance with Ethical Standards:

Ethical Approval: All procedures performed in this study involving human subjects were in accordance with the ethical standards of the US institutional IRB and the Medical Research Council of Zimbabwe, and with the 1964 Helsinki declaration and its later amendments or comparable ethical standards.

Conflict of Interest: Daniel Montaño, Mufuta Tshimanga, Deven Hamilton, Gerald Gorn, and Danuta Kasprzyk each declares that he/she has no conflict of interest.

Informed Consent: Written informed consent was obtained from all individual participants included in the study. 
developing messages to improve demand and maximize parent uptake as IMC programs are rolled out. These targets need to be different among urban and rural expectant mothers and fathers.

\section{Keywords}

Voluntary medical male circumcision (VMMC); Infant circumcision; Integrated Behavioral Model; Evidence based demand creation; Behavior change communication; Implementation science; Behavioral theory

\section{Introduction}

Voluntary Medical Male circumcision (VMMC) programs in sub-Saharan Africa have been in the implementation phase since 2007, after male circumcision was demonstrated to reduce HIV incidence among men by at least $60 \%(1-4)$, and the Joint United Nations Program on HIV/AIDS recommended that VMMC programs be included as part of the overall HIV prevention strategies (5). Since the vast majority of circumcision funding has gone toward training and building VMMC capacity, the expansion of capacity has outpaced demand. VMMC uptake among adult men in sub-Saharan Africa has been challenging. The 14 SubSaharan African priority countries did not achieve their 2015 targets, and Zimbabwe achieved only $27 \%$ of its 2015 goal among the priority target group aged 15 and older (6-8). Zimbabwe is largely a non-circumcising country. The majority Shona and Ndebele ethnic groups have no traditional circumcision practices. Thus, no cultural barriers were anticipated when the Zimbabwe VMMC Program was implemented. Population-based Zimbabwe Demographic and Health Surveys show a 5\% increase of male circumcision prevalence from 9\% in 2010 to $14 \%$ in $2015(9,10)$. This slow VMMC uptake may be due to failure to take into account cultural and behavioral issues, and failure to design evidence-based messaging targeting beliefs that explain VMMC motivation $(11,12)$.

Slow adult VMMC uptake is one factor leading some to recommend increased priority for infant male circumcision (IMC) in sub-Saharan African countries $(11,13)$. Though the public health impact of IMC on HIV incidence will not be seen for two decades, there are a number of advantages of IMC (14). IMC is less expensive and highly cost-effective compared to adult VMMC, and can be integrated into existing health services for infants (13). IMC is safer and easier to perform than adult VMMC, with faster healing and fewer complications $(11,14)$. Surgical devices may further simplify IMC and increase provision by health care providers other than doctors (15-17). IMC reduces lifetime risk of urinary tract infection, and of HIV and several other STIs after sexual debut $(14,18)$. Additionally, risk compensation seems unlikely to be an issue since boys who grow up circumcised will not experience reductions in perceived risk (11).

But how much do we know about parents' attitudes toward IMC? Several study findings suggest that IMC has high acceptability when parents were asked about willingness to consider IMC if it protects their son against HIV (19-23). Several studies have investigated positive and negative issues, besides HIV prevention, that may affect parent motivation to circumcise their infants. Most of these were qualitative or focus group studies that asked participants about willingness to circumcise infants given the protection against HIV, and 
elicited positive and negative opinions about circumcising infants (15, 23-27). Some studies asked about positive reasons only from parents who were interested in IMC, and asked about negative reasons only from those not interested $(23,27)$. None of these studies were able to examine whether the positive and negative issues elicited were actually associated with motivation to circumcise infants. The only studies that have investigated factors associated with IMC motivation examined parent knowledge and demographic characteristics rather than known drivers of behaviors, such as attitudes and beliefs $(21,28)$. In sum, while there has been some research in this area, although not much, the data that has emerged from this research could be considered preliminary. To our knowledge no previous research has examined positive and negative beliefs about IMC, normative influence regarding IMC, perceived environmental facilitators and barriers to IMC, and how these various beliefs affect the willingness of parents to circumcise an infant male. This research begins to fill that void in the literature.

This research was carried out to identify specific mutable key beliefs that best explain parents' level of motivation to have their infant sons circumcised. These key beliefs may serve as the targets for developing messages to improve demand and maximize parent uptake as IMC programs are rolled out.

\section{Methods}

We applied the Integrated Behavioral Model (IBM) as the theoretic framework in this research (see Figure 1) (29). We previously applied this model to identify key beliefs explaining VMMC motivation among adult men and adolescent boys in Zimbabwe (12, 30). The IBM includes constructs from several well established theories, including the Theory of Reasoned Action, Theory of Planned Behavior, Health Belief Model, and Social Cognitive Theory $(31,32)$. Decades of research show that the strongest determinant of behavior is one's motivation or intention to engage in that behavior $(31,33)$. Thus, the IBM focuses on three constructs as determinants of intention: attitude, social influence and personal agency (29). The attitude construct has two components: 1) experiential attitude, one's emotional or affective response to the idea of performing the behavior, and 2) instrumental attitude, cognitively based beliefs about positive or negative consequences or attributes of performing the behavior. Social influence consists of two normative components: 1) beliefs about other's expectations for the person to engage in the behavior (injunctive norm), and 2) beliefs about what others are doing regarding the behavior (descriptive norm). Personal agency consists of two components which impact the ability to engage in the behavior: 1) beliefs about self-efficacy (i.e., perceived ability to overcome obstacles to behavioral performance), and 2) perceived control consisting of beliefs about the effect of facilitators and barriers on behavioral performance. Other factors including a person's characteristics, knowledge, and experiences are considered to impact intention indirectly via these constructs.

The analyses in this paper focus on the five belief-based IBM constructs (instrumental attitude, injunctive norm, descriptive norm, self-efficacy, perceived control) because these are most conducive to change with persuasive messaging in communication campaigns. Our goal was to identify the key beliefs underlying these five constructs, that best explain parent 
IMC motivation. These key beliefs can then be targeted by communication campaigns to change motivation and IMC uptake.

\section{Study Design}

The study included two phases: 1) a questionnaire development phase, and 2) a quantitative survey of a representative sample of expectant mothers and fathers in Zimbabwe. All study procedures were reviewed and approved by the Institutional Review Boards of the US and Zimbabwe collaborating institutions.

\section{Questionnaire Development}

The questionnaire for this study was designed from results of a qualitative elicitation study with expectant parents (29). A sample of 24 expectant mothers and 24 expectant fathers, were recruited through prenatal clinics, about equally divided from four urban and rural areas of Zimbabwe. Expectant fathers were independent samples from the expectant mothers who participated, to increase breadth of response. They participated in interviews to elicit specific issues with respect to each of the IBM constructs: 1) positive and negative beliefs about having their infant son circumcised, 2) sources of normative influence about having their infant son circumcised, and 3) factors that may make it easier or harder to get their infant son circumcised. Interview responses were content analyzed to extract specific issues mentioned with respect to each IBM construct. This resulted in the identification of 52 positive and negative beliefs about having one's infant son circumcised, 21 sources of injunctive and 6 sources of descriptive normative influence, 13 facilitators and 16 constraints.

The quantitative questionnaire was designed based on the qualitative results. Examples of construct measures are shown in Table 1. The 52 positive and negative behavioral beliefs about having one's infant son circumcised were measured on 5-point bipolar agree-disagree scales. Injunctive norm beliefs were measured by asking respondents how strongly they agree or disagree that each of the 21 sources of influence would encourage them to have their infant son circumcised. Descriptive norm beliefs were measured by asking respondents how strongly they agree versus disagree that each of six sources of influence would get their own infant son circumcised. Injunctive and descriptive norm beliefs were each measured on 5-point bipolar agree-disagree scales. Control beliefs were assessed by asking respondents to rate how difficult versus easy each of the 29 facilitators or barriers make it to have their infant son circumcised. These ratings were made on 5-point scales ranging from 'extremely difficult' to 'extremely easy'. Efficacy beliefs were measured by having respondents rate how certain they are that they could get their infant son circumcised if they wanted to, if faced with each barrier. Ratings were made on 5-point scales ranging from 'extremely certain I could not' to 'extremely certain I could'. Finally, intention was measured by asking parents to rate on a 5-point scale how strongly they agree versus disagree that they will get their infant son circumcised if the Ministry of Health and Child Care (MOHCC) began a national program to offer IMC at no cost. This scenario was specified because if and when IMC becomes available, MOHCC will make it free through the National VMMC Program. The questionnaire was translated to Shona and Ndebele, and back-translated to ensure accurate translation of meaning. 


\section{Survey Procedures}

The expectant parent sample for the quantitative survey was recruited via antenatal clinics since the vast majority of pregnant women (93\%) attend antenatal clinics at least 2-3 times during their pregnancy, with $75 \%$ attending 4 or more times (9). Survey respondents were recruited using a three-stage sampling strategy. At the first stage, we selected four geographic areas in Zimbabwe: Harare, Bulawayo, Mutoko District, and Matobo District. Harare and Bulawayo are Zimbabwe's two largest cities, with Harare in the Shona ethnic area and Bulawayo in the Ndebele ethnic area. Mutoko and Matobo Districts are rural areas, with Mutoko being primarily Shona and Matobo being Ndebele.

At the second stage, we selected clinics that provide prenatal care in each of the four study sites. The clinic sampling frame in each urban site consisted of all clinics that provide prenatal care: 18 in Harare, 13 in Bulawayo. In the two rural district sites, the sampling frames were all clinics that provide prenatal care to at least 20 patients per week: 11 in Mutoko, 9 in Matobo. In each of Harare and Bulawayo we randomly selected ten clinics, with probability proportionate to the clinic's average number of prenatal patients seen per week. We used a slightly different clinic selection procedure in the two rural districts due to the large variability in clinic sizes, with a few large hospitals and many small rural clinics. In Mutoko, clinics were grouped into three strata - hospitals, rural health centres, and rural clinics. We selected all three hospitals, one rural health centre, and four rural clinics, totaling 8 prenatal clinics in Mutoko. In Matobo we included all 9 clinics providing prenatal services in the sampling frame (5 hospital clinics, 4 rural clinics).

At the third sampling stage, we recruited expectant parents from the selected clinics, with a goal to independently recruit approximately 200 expectant mothers and 200 expectant fathers from each of the four study geographic sites. Since clinics in Harare and Bulawayo all provide prenatal care to fairly large numbers of patients, we recruited 20 mothers and 20 fathers from each of the ten selected clinics. In the rural districts, where there is much greater variability in prenatal patient volume, we sampled patients proportionate to clinic prenatal patient volume.

After obtaining approval from the Sister-in-Charge of each selected clinic, interviewers coordinated with clinic staff to have expectant mothers over age 18 referred to them. In each clinic, we recruited and surveyed the quota of expectant mothers, followed by recruitment of an independent sample of expectant fathers over age 18. Expectant parents under age 18 or with any previous children who had been circumcised were ineligible. Expectant fathers were recruited via expectant mothers, after ensuring that the mother had not participated in the survey. If the expectant father attended the prenatal visit, the interviewer attempted to recruit him at that time. Otherwise, the interviewer requested the father's contact information from the mother and then attempted to recruit the father. After obtaining informed consent, each face-to-face interview was conducted in a private room in the clinic, or was scheduled for a time and location convenient for the expectant mother or father. Demographic, VMMC knowledge and exposure to VMMC information questions were asked first. Prior to asking IBM questions, interviewers provided an MOHCC description of voluntary medical male circumcision to ensure that participants understood what VMMC is. 
Survey participants selected their preferred language for the survey - English, Shona, or Ndebele.

\section{Analytic Procedures}

Descriptive analyses were carried out on demographic and VMMC knowledge measures. Mean, median, and standard deviation are reported for continuous variables. Percentages are reported for categorical variables.

Our primary analytic goal was to identify IBM measures that best explain IMC motivation. We applied an analytic strategy used and described in prior publications (12,34-37). We first carried out internal consistency analysis and computation of Cronbach's alpha on the questionnaire items used to measure each IBM construct. The construct scores were then computed from their underlying belief items. Before computing instrumental attitude, the behavioral beliefs concerning negative outcomes were reverse coded so that a higher score was associated with greater disagreement that the outcome will occur, implying a more positive attitude. Instrumental attitude toward having an infant son circumcised was then computed as the mean of the 52 behavioral beliefs (Cronbach's a $=.94$ - Expectant Fathers; Cronbach's $a=.95$-Expectant Mothers). Injunctive Norm was computed as the mean of 21 normative beliefs (Cronbach's $a=.97$ - Fathers; Cronbach's $a=.97$ - Mothers). Descriptive Norm was computed as the mean of 6 descriptive norm beliefs (Cronbach's $a=.98$ Fathers; Cronbach's $a=.96$-Mothers). Perceived Control was computed as the mean of the 29 control beliefs (Cronbach's a $=.95$ - Fathers; Cronbach's a $=.93$ - Mothers). SelfEfficacy was computed as the mean of the 16 efficacy beliefs that assessed behavioral certainty under various environmental constraints (Cronbach's a $=.94$ - Fathers; Cronbach's $a=.94$ - Mothers).

The IBM was next applied in three analytic steps, using forward stepwise regression, to explain IMC intention. On the first analytic step, we used the five computed IBM constructs to explain IMC intention. We used forward rather than backward regression because all IBM constructs were significantly correlated with IMC intention, and the goal was to confirm which combination of constructs best explain IMC intention, rather than to carry out exploratory regression. We applied an F-value entry criterion of $p<0.05$ rather than a less strict cutoff because of the substantial power provided by our sample size. The potential impact of clustering by clinic and ethnicity were assessed using mixed effects models and no significant clustering was found.

Analytic steps two and three were carried out with the goal to identify specific beliefs underlying the IBM constructs that best explain IMC motivation, and that therefore may be the best foci for persuasive messages. In analytic step 2 we conducted separate forward stepwise regression analyses for each IBM construct found to be significant in explaining intention in step 1 . The beliefs underlying each significant IBM construct were used as the independent variables. This resulted in identification of the beliefs within each IBM construct that provide significant independent contribution to explaining IMC intention. Since these were separate regression analyses for each IBM construct, the results do not control for variance explained by beliefs across constructs. Thus, analytic step 3 was conducted with beliefs across the IBM constructs. The significant beliefs identified in 
analytic step 2 were combined into a subgroup of beliefs across the IBM constructs, and were included as independent variables in a final stepwise regression analysis to identify beliefs across constructs that provide significant independent explanation of intention. Analytic step 3 resulted in a final model of key beliefs, across the IBM constructs, that best explain IMC intention.

This analytic procedure was carried out separately for the expectant fathers and mothers. We then carried out subgroup analyses of urban and rural fathers and mothers.

\section{Results}

\section{Survey Participants}

Of 939 eligible expectant mothers approached, 56 agreed to participate but did not return to be interviewed after their clinic visit, 44 were too busy, 16 refused, 8 indicated that their partner would not approve, and 15 were unable to participate for some other reason. This resulted in 800 participants equally distributed across the 4 study sites, with an overall participation rate of $85 \%$.

A total of 1,326 expectant mothers were approached to recruit eligible expectant fathers. Of these, 270 indicated that the father was away (179 out of the country, 91 in another part of Zimbabwe) and 17 did not have contact information for the father, leaving 1,039 potential expectant fathers who were contacted. Of these, 91 fathers were too busy to be interviewed, 21 refused, 21 agreed but did not return to be interviewed after the clinic visit, 13 mothers refused participation by the father, and 98 fathers were unable to participate for some other reason. This resulted in 795 expectant fathers surveyed, about equally distributed across the 4 geographic sites, with an overall participation rate of $77 \%$.

Of the 795 expectant fathers and 800 expectant mothers who participated in the survey, 11 of the fathers and 8 of the mothers did not answer the IMC intention question and were excluded from the analysis. Thus, the results presented here are for the remaining 784 fathers and 792 mothers.

\section{Sample Characteristics}

Tables $2 \mathrm{a}$ and $2 \mathrm{~b}$ present demographic and other characteristics of expectant fathers and mothers by study site and total. Expectant fathers were on average 31.6 years of age with 10.6 years of education. Most were married $(96.3 \%)$ and two thirds had at least one other child (66.9\%). Although half of the expectant fathers were selected from Shona areas and half from Ndebele areas of the country, $60.1 \%$ of participants were Shona, 29.6\% Ndebele, and $10.4 \%$ of other ethnicity. The average family income was $\$ 260$ per month, with $93.7 \%$ of the expectant fathers reporting that they regularly earned money including through the informal sector. A vast majority (93.5\%) had correct knowledge that VMMC involves surgical removal of the foreskin, while high percentages were also aware that in some cultures circumcision is an adolescent rite of passage which may include teaching about sex and STI prevention. Nearly $80 \%$ reported having seen promotional information about adult VMMC, with about half (52.3\%) having seen/heard VMMC information on TV or radio, while only about a fifth (18.2\%) had seen such information at health clinics despite a 
relatively high proportion with recent contact with the health clinic setting due to their status as expectant fathers. About a third of expectant fathers (30.2\%) reported that age 0 was the best time to have a boy circumcised.

The expectant mothers were on average 25.3 years of age with 10.3 years of education. Most were married $(87.1 \%)$ with a smaller proportion married in Matobo $(61.3 \%)$, and $60.5 \%$ had at least one child. Ethnicity of mothers was similar to the expectant father sample, with $57.9 \%$ Shona, $35.1 \%$ Ndebele, and $7 \%$ of other ethnicity. The average monthly family income was $\$ 243$, with $49.9 \%$ of mothers reporting that they regularly earned money. Four fifths $(82.8 \%)$ knew that VMMC involves surgical removal of the foreskin, while nearly half knew circumcision is a rite of passage in some cultures and two thirds thought it can include teaching adolescents about sex and STI prevention. Nearly two thirds (63.2\%) reported having seen promotional information about VMMC, with just under half (43.3\%) having seen/heard VMMC information on TV or radio, while only $13.6 \%$ had seen such information at health clinics despite being recruited in an antenatal health clinic. Nearly a third (31.6\%) of the expectant mothers also reported that age 0 was the best time to have a boy circumcised.

\section{Multiple Regression: Fathers}

On the first step of regression analyses for expecting fathers, intention to get their infant son circumcised was significantly explained $(R=.70, p<.001)$ by instrumental attitude $(\beta=$. $34, p<.001)$, descriptive norm $(\beta=.21, p<.002)$, and self-efficacy $(\beta=.25, p<.001)$ (Table 3). This suggests that interventions targeting these three constructs may be most effective to encourage IMC uptake.

In analytic step two, three separate stepwise regression analyses were carried out with the beliefs underlying instrumental attitude, descriptive norm, and self-efficacy as the independent variables. Table 4 (first column) lists the beliefs entering each of these regressions along with their beta coefficients. Note that in these and other results, some variables reversed their beta coefficients late in the regression entry, thus acting as suppressors with coefficient direction opposite to what one would expect. Additionally, only the significant items are listed in Table 4.

Behavioral Beliefs-All but two of the 52 behavioral beliefs were significantly correlated with IMC intention. The stepwise regression resulted in 15 behavioral beliefs ( 5 positive, 10 negative) entering the equation to explain IMC intention $(R=.70)$. Results indicate that fathers who are motivated believe that healing will be fast with low likelihood of complications, and it will protect the baby for the future. By contrast, fathers' concerns focus on the infant being too young for the pain, disfigurement and impact on growth, and having the decision taken away from the child and blaming the parent.

Descriptive Norm Beliefs-All six descriptive norm beliefs were significantly correlated with intention. Five beliefs concerned with whether closest friends, brothers, other relatives, people like them, and parents of infants in their community would have their infant circumcised, entered the regression equation to explain IMC intention $(R=.60)$. 
Efficacy Beliefs-All 16 efficacy beliefs were significantly correlated with IMC intention, with six beliefs entering the stepwise regression $(R=.64)$. These results suggest that fathers' efficacy for IMC could be enhanced if services were local, if they are told how IMC reduces risk of infection, if messages are focused on assuring fathers that clinics are adequately supplied for IMC, encouraging fathers to get circumcised, and reducing perceptions that culture is against circumcision and that it is painful.

Final Regression Model-The first column of Table 5 lists the results of the third analytic step for fathers. Eleven behavioral beliefs, one descriptive norm belief, and four efficacy beliefs independently explained IMC intention $(R=.76)$. This final model for expectant fathers identified specific beliefs that might be targeted using a mass media approach. Fathers' concerns about the infant being too young, disfigurement, not growing properly, and blaming his parents will need to be addressed, along with efficacy concerns such as adequacy of clinic supplies, perception that culture may be against it, and not knowing how IMC prevents HIV. Positive beliefs about low likelihood of complications and lifelong protection should be reinforced, along with the perception that closest friends would have their infant sons circumcised.

\section{Multiple Regression: Mothers}

Mother's IMC intention was significantly explained $(R=.72, p<.001)$ by instrumental attitude $(\beta=.23, p<.001)$, injunctive norm $(\beta=.18, p=.001)$, descriptive norm $(\beta=.17, p$ $<.001)$, self-efficacy $(\beta=.16, p<.001)$, and perceived control $(\beta=.13, p=.004)$. This suggests that interventions may be most effective if they target all five IBM model constructs to increase expectant mothers' IMC intentions (Table 3).

In analytic step two, regression analyses were carried out with the beliefs underlying each of the five model constructs. The second column of Table 4 lists the results.

Behavioral Beliefs-All but two of the 52 behavioral beliefs were significantly correlated with IMC intention. In the stepwise regression, 15 behavioral beliefs (6 positive, 9 negative) entered to explain expectant mothers' IMC intention $(R=.74)$. Six of these beliefs were also significant for fathers, while nine beliefs were unique to expectant mothers. Mothers who are motivated believe they are doing it for the child and showing their love, and it would be hygienic. Less motivated mothers are more likely to believe it would be against her religion, his penis will be too small so things might go wrong disabling him, it will be seen as child abuse, and she will have forced her will on him when he is too young to make the decision himself.

Injunctive Norm Beliefs-All 21 injunctive norm beliefs were significantly correlated with IMC intention, with 5 entering the regression analysis $(R=.65)$. Mothers with higher IMC intention were more likely to perceive support from the infant's father, family, health care workers in the community, other parents of infants, and her religion/church.

Descriptive Norm Beliefs-All six descriptive norm beliefs were significantly correlated with IMC intention, with three entering the regression equation $(R=.60)$. Mothers with 
higher IMC intention were more likely to believe that people like them, closest friends, and their sisters would circumcise their infant boys.

Efficacy Beliefs-All 16 efficacy beliefs were significantly correlated with IMC intention, and four entered the stepwise regression $(R=.67)$. These results suggest that mothers' efficacy for IMC could be increased if it is provided in local (including rural) clinics, and if messages reduced mothers' perceptions that it is painful and that culture is against it, and affirmed that IMC is important to do even though it is new in the community.

Control Beliefs-All 29 perceived control beliefs were significantly correlated with IMC intention. Seven beliefs (4 facilitators, 3 barriers) entered the stepwise regression to explain IMC intention $(R=.64)$. These results suggest that IMC motivation among expectant mothers can be increased through messages that explain how IMC prevents HIV infection, local chiefs supporting IMC, making IMC available in local clinics, and providing transportation.

Final Regression Model-The second column of Table 5 shows the results from the third analytic step for mothers. Ten behavioral beliefs, one injunctive norm, one descriptive norm, two efficacy beliefs, and two control beliefs significantly explain expectant mothers' IMC intention $(R=.78)$. This final model suggests the need to tailor mass media communication to increase IMC motivation differently for mothers than for fathers. Though they should be similar with respect to reinforcing positive beliefs about IMC having few complications and growing up protected, many other key target beliefs are different for mothers. Mothers' beliefs about IMC having few complications and growing up protected need to be reinforced, along with the perceptions that closest friends would circumcise their infant sons, and that the father supports IMC. Mothers' concerns that IMC is difficult and may disable the infant, it will be viewed as child abuse, is forcing her will on him, and is against her religion will need to be addressed, along with efficacy concerns of IMC availability in local clinics and not having been available before. Transportation and lack of knowledge about how circumcision prevents HIV also appear to be important barriers for mothers.

\section{Multiple Regression: Urban/Rural Subgroups}

The above regression analysis procedures were next conducted separately for urban and rural fathers and mothers. In the first analytic step (Table 3), instrumental attitude, descriptive norm, and self-efficacy significantly explained IMC intention among both urban $(R=.67)$ and rural $(R=.74)$ fathers. Urban mothers' IMC intention was significantly explained $(R=.68)$ by instrumental attitude, injunctive norm, and self-efficacy, while rural mothers' IMC intention was explained $(R=.76)$ by all five IBM constructs.

Results from the second analytic step of regression analyses are described below, organized by IBM construct (Table 4).

Behavioral Beliefs-Twelve behavioral beliefs significantly explained IMC intention among both urban fathers $(R=.71)$ and rural fathers $(R=.78)$. Three of these beliefs were common for both urban and rural fathers (prepare him for life ahead, possible disfigurement, and the need to wait to see if he will live longer than 3 years), while the other nine beliefs for 
each group were different. Among urban mothers, seven behavioral beliefs significantly explained IMC intention ( $R=.68$ ), while 11 behavioral beliefs were significant for rural mothers $(R=.79)$. The beliefs that it is against our religion, and you would be doing it for the child and showing your love, were the only two beliefs in common for urban and rural mothers. Comparing urban father and mother results, there were only two significant beliefs in common. There were five (fewer than 50\%) common significant beliefs among rural fathers and mothers. These results suggest the need to target very different behavioral beliefs in order to change instrumental attitude toward IMC among these four subgroups of expectant parents.

Injunctive Norm Beliefs-Injunctive norm belief analyses were conducted only for mothers, as this IBM construct was not significant among fathers in analytic step one. Urban mothers' IMC intention was significantly explained $(R=.65)$ by six injunctive norm beliefs, while rural mothers' IMC intention was explained by 3 injunctive norm beliefs $(R=.68)$. Only normative influence from the child's father was common for urban and rural mothers, suggesting that interventions targeting normative support need to target different sources of influence for these subgroups of expectant mothers.

Descriptive Norm Beliefs-Two descriptive norm beliefs explained IMC intention among urban fathers $(R=.55)$ and rural fathers $(R=.64)$, while three descriptive norm beliefs were significant for rural mothers $(R=.65)$. Only the belief regarding most people like you, was common for all three subgroups. Urban fathers were also influenced by whether other relatives would have their infant sons circumcised, while rural fathers were influenced by brothers, and rural mothers were influenced by whether closest friends and sisters would circumcise their infant sons.

Efficacy Beliefs-Four efficacy beliefs were significant predictors of IMC intention among urban fathers $(R=.56)$, while five beliefs were significant for rural fathers $(R=.71)$. Only one belief (if MC is available in local clinics) was common for urban and rural fathers. Three efficacy beliefs were significant in explaining IMC intention among urban mothers $(R$ $=.63)$, while four efficacy beliefs were significant among rural mothers $(R=.72)$. Two efficacy beliefs concerned with people describing IMC as painful and availability in local clinics, were common among urban and rural mothers. Only the efficacy belief concerning availability in local clinics was common for all four subgroups. This lack of commonality of beliefs suggests the need to develop different communication strategies for the subgroups to increase self-efficacy for IMC with respect to different barriers. Besides the common beliefs, efficacy with respect to the mother being against it, perceived shortage of trained staff, and not offering it previously, need to be addressed for urban fathers, while not being circumcised, worry about supplies, and culture being against it need to be addressed for rural fathers. Perception of culture being against IMC is important for urban mothers, while efficacy beliefs about not knowing where to go, and IMC not being offered before, need to be addressed for rural mothers.

Control Beliefs-Regression analysis of control beliefs was conducted only among rural mothers. Six control beliefs ( 3 facilitators, 3 barriers) were significant in explaining IMC 
intention $(R=.71)$. Results indicate the need to reinforce media promotion and support by chiefs, provide transportation, and address barriers concerned with mothers not knowing how IMC prevents HIV, people describing it as painful, and availability only in city clinics.

Final Regression Model-Results of the third analytic step for each of the four subgroups are shown in Table 5. The final model for urban fathers included 10 behavioral beliefs, one descriptive norm belief, and one efficacy belief $(R=.72)$. IMC motivation for this group was affected by beliefs including healing, preparation for life ahead, protection, pain exposure, disfigurement, ability to have children, blaming the parents, and culture. These findings indicate that behavior change messages for urban fathers may be most effective if they primarily target behavioral beliefs. IMC intention among rural fathers was significantly explained $(R=.81)$ by 8 behavioral beliefs, one descriptive norm belief, and 4 efficacy beliefs. Only three of these beliefs (preparation for life ahead, possible disfigurement, wait to see if he will live longer than 3 years) were also significant for urban fathers. The findings for rural fathers suggest that behavior change messages should primarily target different behavioral and efficacy beliefs than for urban fathers.

The final model for urban mothers found IMC motivation was significantly explained by four behavioral beliefs, five injunctive norm beliefs, and two efficacy beliefs $(R=.74)$. Only one behavioral belief (too young for pain exposure) was also significant for urban fathers, while urban mothers' motivation is also affected by contrasting beliefs about doing it for the child and concern that IMC may be viewed as child abuse. These results suggest that an intervention to increase IMC motivation among urban mothers should focus on perceived normative support from a range of influence sources, as well as several behavioral beliefs. IMC intention among rural mothers was significantly explained by seven behavioral beliefs, one descriptive norm belief, two efficacy beliefs, and one control belief $(R=.83)$. Only one behavioral belief (doing it for the child) was also significant for urban mothers, while three were also significant for fathers. In contrast to urban mothers, rural mothers' IMC intention is more strongly influenced by behavioral beliefs, while perception of what sisters would do, media promotion, and efficacy beliefs with respect IMC availability were also important. Only two of the 11 final model beliefs for rural mothers were significant for urban mothers, indicating that targets for behavior change communication need to be quite different for rural versus urban mothers.

\section{Discussion}

This is the first study that we are aware of to investigate positive and negative beliefs, and to assess their strength of association with IMC motivation. An important strength of this study is that the measures and research were driven by strong behavioral theory of how beliefs determine attitude, social influence and personal agency constructs. Thus, in addition to identifying constructs that are important determinants of IMC motivation, our goal was to identify specific key beliefs that explain motivation. It is these beliefs that can serve as foci for messages to change motivation.

The patterns of the significant belief results have important implications for communication campaigns. Beliefs that were commonly significant in the final model for mothers and 
fathers may serve as targets for messaging in a mass media campaign. For example, messages for both mothers and fathers should reinforce the positive behavioral beliefs that IMC is less likely to have complications than if the child is older, and results in the boy growing up protected. Messages should counter the concern that it may not heal properly and cause disfigurement. Similarly, messages should reinforce the descriptive norm belief that closest friends would circumcise their infants. Importantly, perceived efficacy with respect to IMC availability in local clinics was significant for both mothers and fathers, particularly rural expectant parents. The adult VMMC program began as a vertical system with dedicated VMMC clinics in only a few locations. The fact that local access was a significant issue for both mothers and fathers is likely a reaction to this. Thus, it will be important for IMC to be available in local clinics, including at rural clinics so that transportation will not be a barrier. Incorporating IMC into maternal-child health services would use an already existing infrastructure.

Our results also show some important differences between mothers and fathers with respect to significant beliefs explaining IMC motivation. In the final model for mothers and fathers there were 8 additional significant behavioral beliefs for fathers and 7 additional significant behavioral beliefs among mothers. In the final models, behavioral beliefs specifically significant for mothers were concerned with the infant's penis being too small so something may go wrong and disable him, and several beliefs related to how she may be viewed if she were to have her son circumcised. These included negative beliefs that it would be seen as child abuse, and you will have forced your will on him, as well as the positive belief that you would be doing it for the child and showing your love. By contrast, significant behavioral beliefs specific to fathers included negative and positive beliefs concerned with the child's age (disturbing his growth to be a full man, should not be done until we know he will live longer than 3 years, prepare him for the life ahead, allows ample time for penis to become strong), and concern that the child will blame his parents for making the decision. These results suggest that it is important for mass media messaging to target different beliefs for expectant mothers and fathers. Thus, although there should be some common messages, a campaign would also need to include messages specifically directed to mothers and different messages directed to fathers, and care would need to be taken to ensure that these messages are complementary.

In addition, we found important differences in determinants of IMC motivation between urban and rural expectant parents. Among urban fathers, IMC motivation was explained almost entirely by behavioral beliefs in the final model. By contrast rural fathers' motivation was explained by both behavioral and efficacy beliefs. Only three of the behavioral beliefs were significant for both urban and rural fathers, while 7 additional beliefs were significant among urban and 5 different beliefs were significant among rural fathers. These findings indicate that different sets of behavioral beliefs should be targeted in motivational messages for urban versus rural fathers, and efficacy beliefs should also be targeted in messages for rural fathers.

Among urban mothers, behavioral and normative beliefs were about equally represented in the final model, with two efficacy beliefs also entering. By contrast, IMC motivation among rural mothers is mostly explained by behavioral beliefs, with one or two descriptive norm, 
efficacy and control beliefs also entering. These findings suggest that communication messages for urban expectant mothers need to equally target behavioral beliefs and normative beliefs about perceived support from several sources (child's father, grandparents, health care workers, church, culture). By contrast, messages for rural mothers should primarily target behavioral beliefs. Additionally, our final models indicate that, with the exception of one belief (doing it for the child and showing your love), different sets of behavioral beliefs need to be targeted to increase IMC motivation among urban versus rural expectant mothers.

Our research has identified numerous key beliefs associated with IMC motivation among expectant parents, including different sets of beliefs for urban and rural mothers and fathers. It is important to note that the beliefs identified in our final models should not necessarily be the only targets for communication messages. Indeed, other beliefs that were strongly correlated with IMC motivation may not have entered the stepwise regressions due to collinearity with beliefs that had already entered the model. These beliefs should also be considered as potential targets for intervention, particularly if they are more amenable to change through communication messages. Communication campaigns will need to target multiple key beliefs that were identified in our analyses, as well as other beliefs that are highly correlated with a target belief that may not lend itself as easily to change.

There are a few possible limitations to this study. First, it is important to note that in most of the models presented, at least one belief entered the model as a suppressor with beta coefficients having the opposite sign than one would expect. This is due to the high level of correlation between the beliefs. The overall impact of multicollinearity on interpretation in this context is negligible because we are not concerned with specific interpretations of the beta coefficients or with informing future predictive models, rather we are interested in the set of beliefs which collectively account for the variance in IMC intention.

A second limitation is the possibility that our sample of expectant fathers may not be representative given the high proportion (94\%) who reported they regularly earn money. This is certainly higher than our household based sample of men where $65 \%$ reported regularly earning money (12). However, this finding is not surprising for several reasons. When answering the income question, men included informal income such as trading and subsistence farming. Two-thirds of expectant fathers had at least one other child, so they were likely highly motivated to earn money, and with a mean age of 32 they were in their prime earning years compared with a mean age of 23 among our household-based sample of men. Given these factors, along with the expectant father survey participation rate of $77 \%$, we are confident that this sample is representative of expectant fathers in Zimbabwe.

A third potential limitation is that we have assumed that increased IMC intention will result in increased likelihood of IMC uptake among expectant parents. We do know that intention is the best predictor of behavior (31). Indeed, a systematic review followed by a metaanalysis of diverse behavioral domains showed a mean correlation of 0.53 between intention and behavior $(38,39)$. We do not know what level of increased motivation is required to tip someone into action. With respect to IMC, this is likely to vary for different individuals and circumstances, and should be the focus of additional longitudinal research of the effect of 
motivation on uptake of circumcision. A fourth limitation of this study is that the results may be specific to Zimbabwe. The key beliefs we identified may not be generalizable to other countries. However, the strength of this research is that it provides a theory-driven framework and strong methodology that can be applied in other countries to identify evidence-based target beliefs specific to those settings.

We have identified key beliefs that best explain parent motivation for IMC. These beliefs should be targeted through careful design of persuasive communications in order to increase parent motivation and uptake of IMC. Zimbabwe and other VMMC priority countries are considering IMC in addition to adolescent and adult VMMC as part of their HIV prevention programs. In the adult VMMC programs, it was generally assumed that building the infrastructure, training clinicians, making VMMC available, and focusing messages on the HIV prevention aspects of VMMC would be sufficient to produce demand. Slow uptake among adult men has led to the need to develop evidence-based motivational strategies and messaging to increase uptake. As IMC programs begin to be implemented, we strongly recommend that careful communication design be done right from the beginning in order to maximize parent motivation when IMC becomes available. Our study results provide the foundation for designing evidence-based messaging to change parent beliefs, attitudes, and motivation for IMC. By applying evidence based demand creation focused on expectant parents in conjunction with a transition towards increased capacity for IMC, program implementers will hopefully avoid many of the current difficulties found in the adult VMMC programs with capacity far outpacing demand. This work represents the first step in developing an evidence-based demand creation program for IMC in Zimbabwe. The next step will be to translate these beliefs into specific messages that can be tested and refined before they are implemented on a large scale.

\section{Acknowledgments}

The research reported in this publication was supported by the National Institute of Mental Health of the National Institutes of Health under Award Number R01MH083594. The content is solely the responsibility of the authors and does not necessarily represent the official views of the National Institutes of Health. We would like to acknowledge the study participants and the University of Zimbabwe, Department of Community Medicine ZiCHIRe Program study team for their enthusiastic participation in all phases of this study.

\section{References}

1. Auvert B, Taljaard D, Lagarde E, Sobngwi-Tambekou J, Sitta R, Puren A. Randomized, controlled intervention trial of male circumcision for reduction of HIV infection risk: the ANRS 1265 Trial. PLoS medicine. 2005; 2(11):e298. [PubMed: 16231970]

2. Bailey RC, Moses S, Parker CB, Agot K, Maclean I, Krieger JN, et al. Male circumcision for HIV prevention in young men in Kisumu, Kenya: a randomised controlled trial. Lancet. 2007; 369(9562):643-56. [PubMed: 17321310]

3. Byakika-Tusiime J. Circumcision and HIV infection: assessment of causality. AIDS and behavior. 2008; 12(6):835-41. [PubMed: 18800244]

4. Gray RH, Kigozi G, Serwadda D, Makumbi F, Watya S, Nalugoda F, et al. Male circumcision for HIV prevention in men in Rakai, Uganda: a randomised trial. Lancet. 2007; 369(9562):657-66. [PubMed: 17321311]

5. WHO, UNAIDS. New Data on Male Circumcision and HIV Prevention: Policy and Programme Implicationz. Geneva: UNAIDS; 2007. 
6. Awad SF, Sgaier SK, Ncube G, Xaba S, Mugurungi OM, Mhangara MM, et al. A Reevaluation of the Voluntary Medical Male Circumcision Scale-Up Plan in Zimbabwe. PloS one. 2015; 10(11):e0140818. [PubMed: 26529596]

7. MOHCC. Global AIDS Response Report. Geneva, Switzerland: Ministry of Health and Child Care; 2016.

8. UNAIDS. Prevention Gap Report. 20 Avenue Appia CH-1211 Geneva 27. Switzerland: United Nations Programme on HIV/AIDS; 2016.

9. ZDHS. Zimbabwe Demographic and Health Survey 2010-11. Calverton, Maryland: 2012.

10. ZDHS. Zimbabwe Demographic and Health Survey: Key Indicators. Rockville, Maryland; Harare, Zimabwe: The DHS Program, ICF International; Zimbabwe National Statistics Agency (ZIMSTAT); 2016.

11. Kalichman SC. Neonatal circumcision for HIV prevention: Cost, culture, and behavioral considerations. PLoS medicine. 2010; 7(1):e1000219. [PubMed: 20098515]

12. Montano DE, Kasprzyk D, Hamilton DT, Tshimanga M, Gorn G. Evidence-based identification of key beliefs explaining adult male circumcision motivation in Zimbabwe: targets for behavior change messaging. AIDS and behavior. 2014; 18(5):885-904. [PubMed: 24443147]

13. Binagwaho A, Pegurri E, Muita J, Bertozzi S. Male circumcision at different ages in Rwanda: a cost-effectiveness study. PLoS medicine. 2010; 7(1):e1000211. [PubMed: 20098721]

14. Morris BJ, Waskett JH, Banerjee J, Wamai RG, Tobian AA, Gray RH, et al. A 'snip' in time: what is the best age to circumcise? BMC Pediatr. 2012; 12:20. [PubMed: 22373281]

15. Mavhu W, Hatzold K, Laver SM, Sherman J, Tengende BR, Mangenah C, et al. Acceptability of early infant male circumcision as an HIV prevention intervention in Zimbabwe: a qualitative perspective. PloS one. 2012; 7(2):e32475. [PubMed: 22384258]

16. Mavhu W, Larke N, Hatzold K, Ncube G, Weiss HA, Mangenah C, et al. Implementation and Operational Research: A Randomized Noninferiority Trial of AccuCirc Device Versus Mogen Clamp for Early Infant Male Circumcision in Zimbabwe. J Acquir Immune Defic Syndr. 2015; 69(5):e156-63. [PubMed: 26010029]

17. Sinkey RG, Eschenbacher MA, Walsh PM, Doerger RG, Lambers DS, Sibai BM, et al. The GoMo study: a randomized clinical trial assessing neonatal pain with Gomco vs Mogen clamp circumcision. Am J Obstet Gynecol. 2015; 212(5):664, e1-8. [PubMed: 25794628]

18. Morris BJ, Wiswell TE. Circumcision and lifetime risk of urinary tract infection: a systematic review and meta-analysis. J Urol. 2013; 189(6):2118-24. [PubMed: 23201382]

19. Albert LM, Akol A, L'Engle K, Tolley EE, Ramirez CB, Opio A, et al. Acceptability of male circumcision for prevention of HIV infection among men and women in Uganda. AIDS care. 2011; 23(12):1578-85. [PubMed: 21732902]

20. Mavhu W, Buzdugan R, Langhaug LF, Hatzold K, Benedikt C, Sherman J, et al. Prevalence and factors associated with knowledge of and willingness for male circumcision in rural Zimbabwe. Tropical medicine \& international health : TM \& IH. 2011; 16(5):589-97. [PubMed: 21349135]

21. Mugwanya KK, Whalen C, Celum C, Nakku-Joloba E, Katabira E, Baeten JM. Circumcision of male children for reduction of future risk for HIV: acceptability among HIV serodiscordant couples in Kampala, Uganda. PloS one. 2011; 6(7):e22254. [PubMed: 21799805]

22. Plank RM, Makhema J, Kebaabetswe P, Hussein F, Lesetedi C, Halperin D, et al. Acceptability of infant male circumcision as part of HIV prevention and male reproductive health efforts in Gaborone, Botswana, and surrounding areas. AIDS and behavior. 2010; 14(5):1198-202. [PubMed: 19924526]

23. Rediger C, Muller AJ. Parents' rationale for male circumcision. Can Fam Physician. 2013; 59(2):e110-5. [PubMed: 23418252]

24. Jarrett P, Kliner M, Walley J. Early infant male circumcision for human immunodeficiency virus prevention: knowledge and attitudes of women attending a rural hospital in Swaziland, Southern Africa. SAHARA J. 2014; 11:61-6. [PubMed: 24957082]

25. Mavhu W, Mupambireyi Z, Hart G, Cowan FM. Factors associated with parental non-adoption of infant male circumcision for HIV prevention in Sub-Saharan Africa: a systematic review and thematic synthesis. AIDS and behavior. 2014; 18(9):1776-84. [PubMed: 24980247] 
26. Waters E, Stringer E, Mugisa B, Temba S, Bowa K, Linyama D. Acceptability of neonatal male circumcision in Lusaka, Zambia. AIDS care. 2012; 24(1):12-9. [PubMed: 21711163]

27. Young MR, Odoyo-June E, Nordstrom SK, Irwin TE, Ongong'a DO, Ochomo B, et al. Factors associated with uptake of infant male circumcision for HIV prevention in western Kenya. Pediatrics. 2012; 130(1):e175-82. [PubMed: 22711723]

28. Waters E, Li M, Mugisa B, Bowa K, Linyama D, Stringer E, et al. Acceptability and uptake of neonatal male circumcision in Lusaka, Zambia. AIDS and behavior. 2013; 17(6):2114-22. [PubMed: 22968397]

29. Montano, DE., Kasprzyk, D. Theory of Reasoned Action, Theory of Planned Behavior, and the Integrated Behavioral Model. In: Glanz, K.Rimer, BK., Viswanath, K., editors. Health Behavior: Theory, Research, and Practice. 5. San Francisco, CA: Jossey-Bass; 2015. p. 95-124.

30. Kasprzyk D, Tshimanga M, Hamilton DT, Gorn GJ, Montano DE. Identification of Key Beliefs Explaining Male Circumcision Motivation Among Adolescent Boys in Zimbabwe: Targets for Behavior Change Communication. AIDS and behavior. 2017 Jan 12. Published online.

31. Fishbein, M., Ajzen, I. Predicting and Changing Behavior: The Reasoned Action Approach. New York, NY: Psychology Press, Taylor \& Francis Group; 2010.

32. Bandura, A. Social cognitive theory and control over HIV infection. In: DiClemente, RJ., Peterson, JL., editors. Preventing AIDS: Theories and methods of behavioral interventions. New York, NY: Plenum Press; 1994. p. 25-59.

33. Ajzen, I. Martin Fishbein's Legacy: The Reasoned Action Approach. In: Hennessy, M., editor. The Annals of The American Academy of Political and Social Science. Vol. 640. Thousand Oaks, CA: Sage; 2012. p. 11-27.

34. Bleakley, A., Hennessy, M. The Quantitative Analysis of Reasoned Action Theory. In: Hennessy, M., editor. The Annals of The American Academy of Political and Social Science. Vol. 640. Thousand Oaks, CA: Sage; 2012. p. 28-41.

35. Fishbein M, Cappella JN. The role of theory in developing effective health communications. Journal of Communication. 2006; 56:S1-S17.

36. Kasprzyk, D., Montano, DE. Application of an integrated behavioral model to understand HIV prevention behavior of high-risk men in rural Zimbabwe. In: Ajzen, I.Albarracin, D., Hornik, R., editors. Prediction and Change of Health Behavior: Applying the Reasoned Action Approach. Hillsdale, NJ: Lawrence Erlbaum Associates, Inc; 2007.

37. von Haeften I, Fishbein M, Kasprzyk D, Montano D. Analyzing data to obtain information to design targeted interventions. Psychology, Health and medicine. 2001 May; 6(2):151-64.

38. Sheeran, P. Intention-behavior relations: A conceptual and empirical review. In: Stroebe, W., Hewstone, M., editors. European review of social psychology. Chichester, UK: Wiley; 2002.

39. Webb TL, Sheeran P. Does changing behavioral intentions engender behavior change? A metaanalysis of the experimental evidence. Psychological bulletin. 2006; 132(2):249-68. [PubMed: 16536643] 


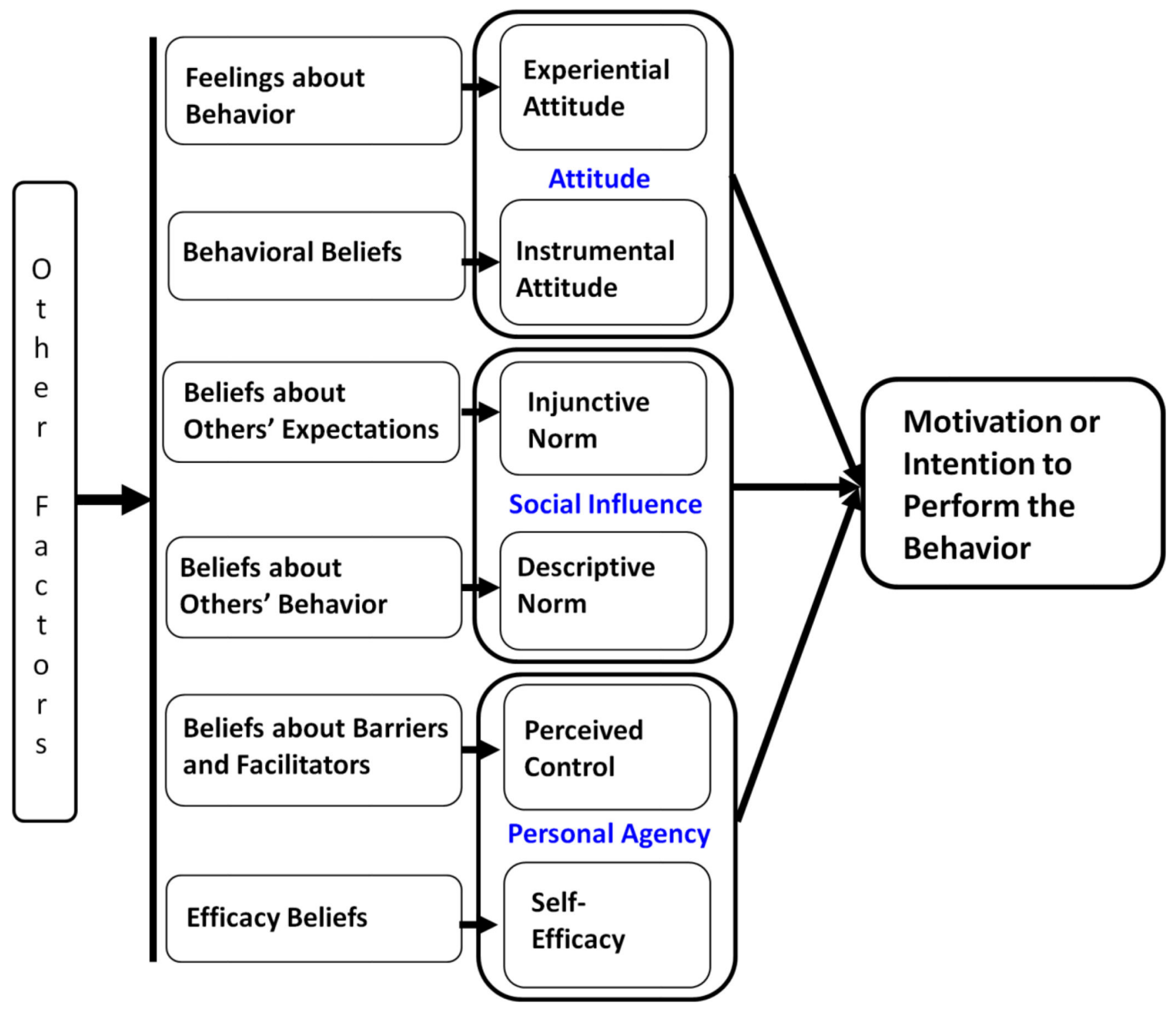

Figure 1.

Integrated Behavioral Model 


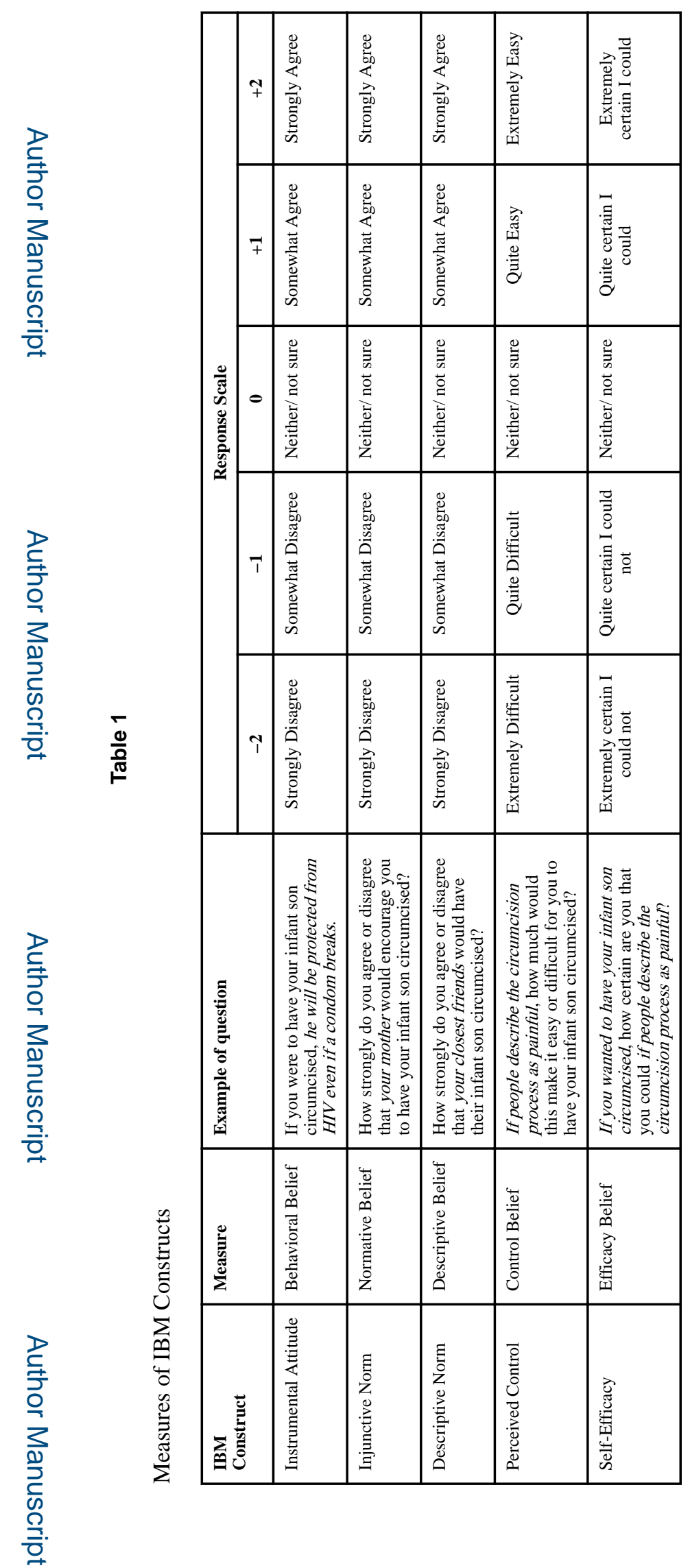

AIDS Behav. Author manuscript; available in PMC 2019 February 01. 


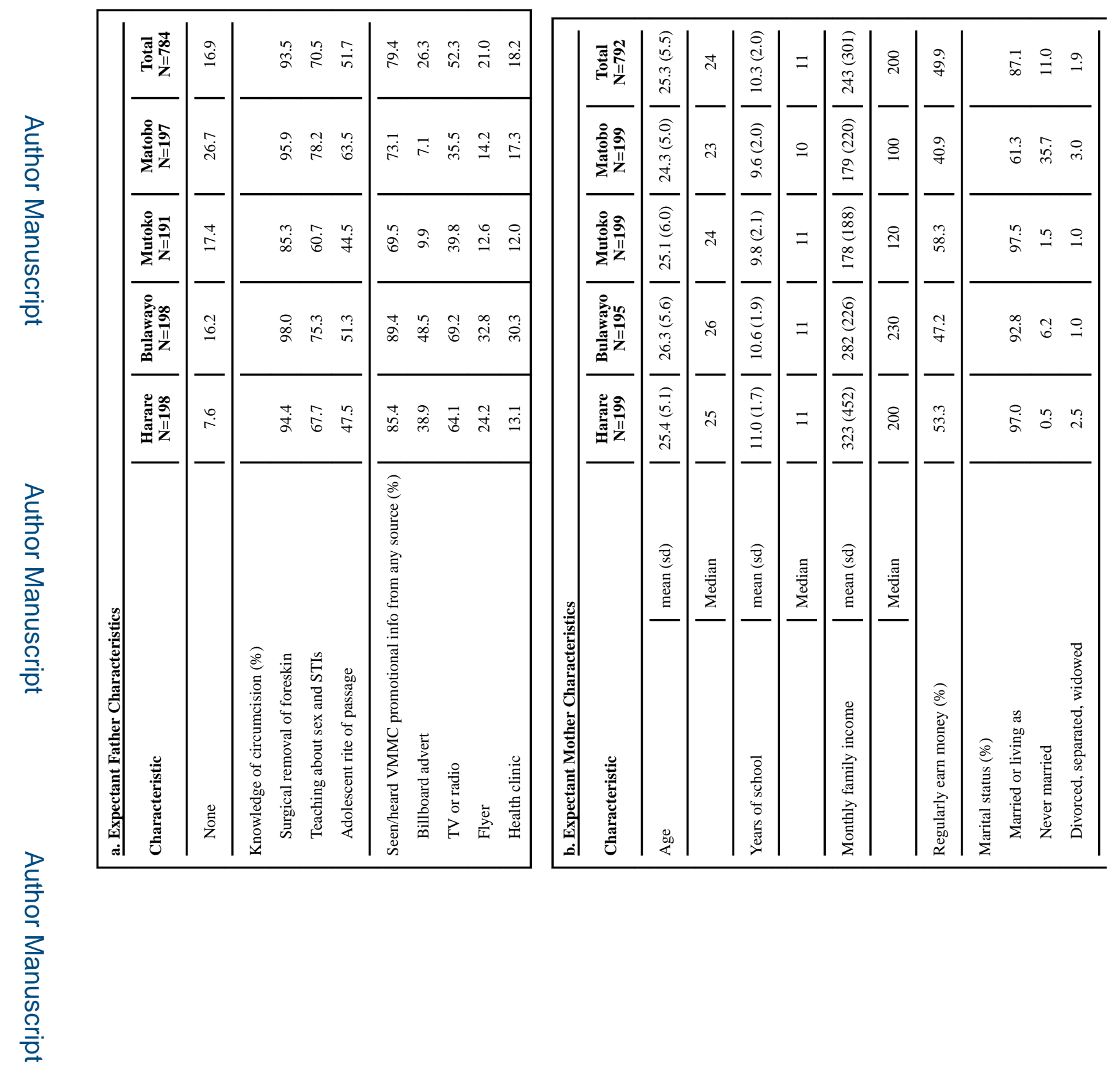

로을 


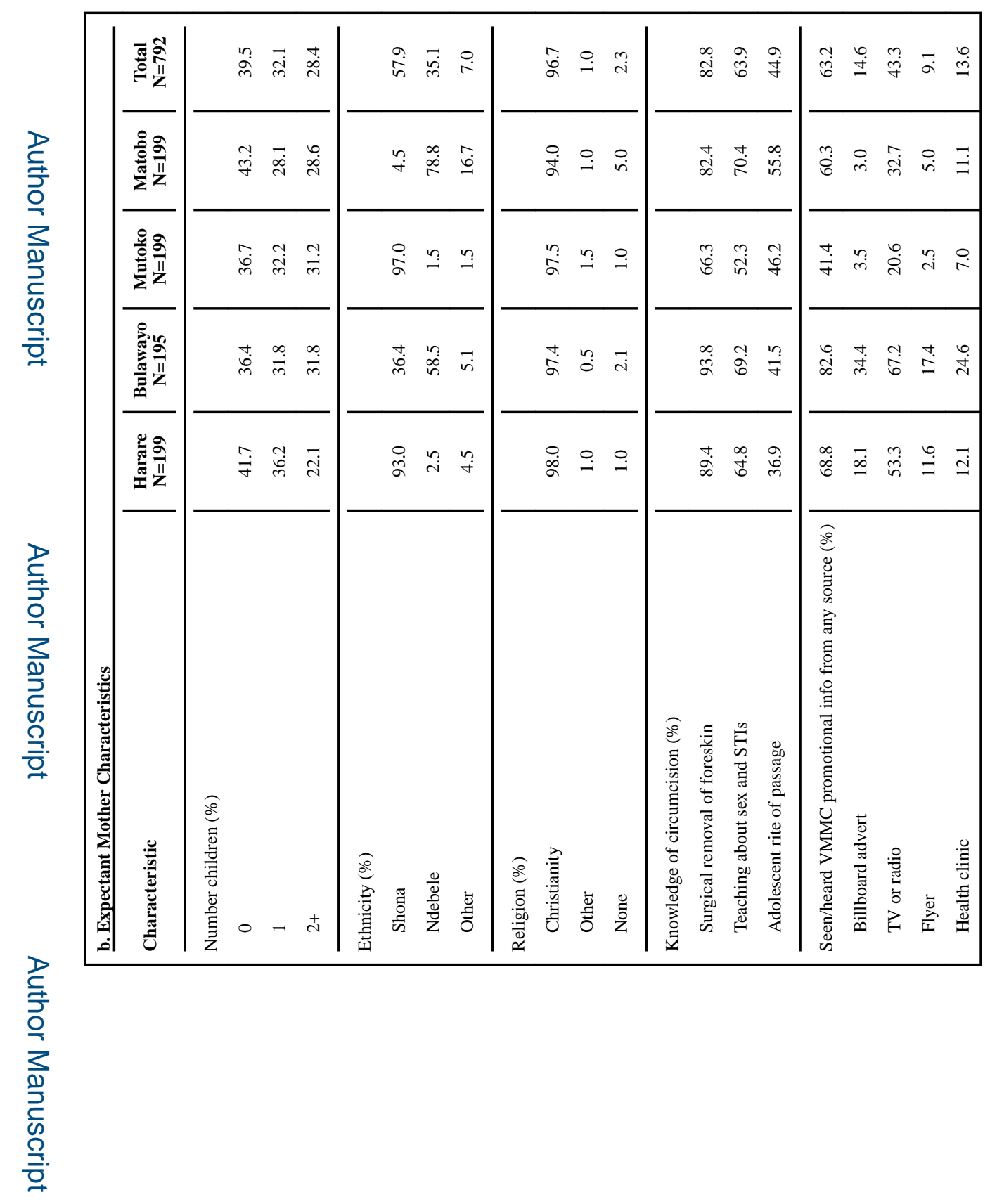

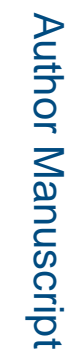




\begin{tabular}{|c|c|c|c|c|c|c|}
\hline 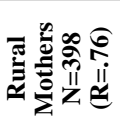 & $\begin{array}{l}\hat{3} \\
a\end{array}$ & 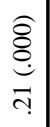 & 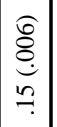 & 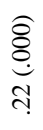 & 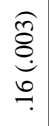 & 畩 \\
\hline 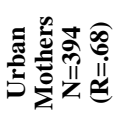 & $\begin{array}{l}\hat{a} \\
a\end{array}$ & 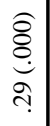 & 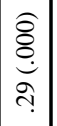 & $\tilde{z}$ & 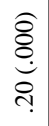 & z \\
\hline 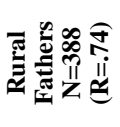 & 言 & 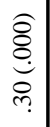 & $\bar{z}$ & & 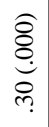 & ż \\
\hline 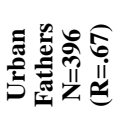 & $\widehat{\Xi}$ & 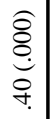 & $\tilde{z}$ & 宅 & 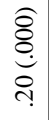 & z \\
\hline 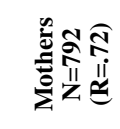 & $\begin{array}{l}\hat{a} \\
a\end{array}$ & 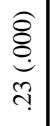 & 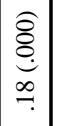 & 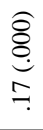 & 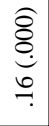 & $\begin{array}{l}\stackrel{f}{8} \\
\stackrel{8}{\oplus} \\
?\end{array}$ \\
\hline 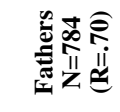 & 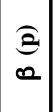 & 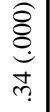 & $\tilde{z}$ & 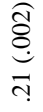 & 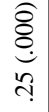 & z \\
\hline & 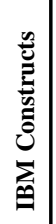 & 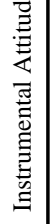 & 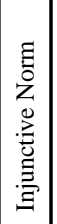 & 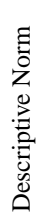 & 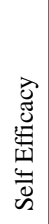 & 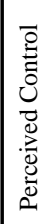 \\
\hline
\end{tabular}




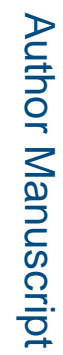

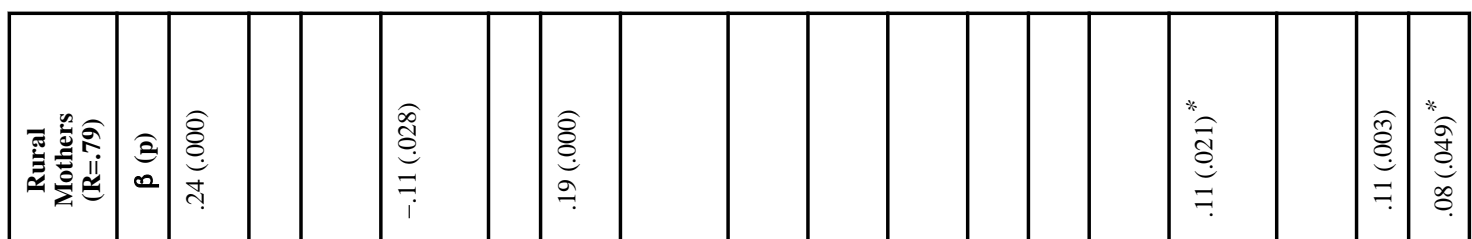

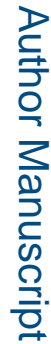

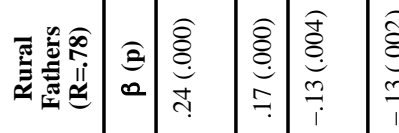

ב

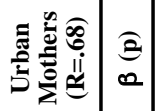

$\stackrel{+}{\frac{0}{6}}$

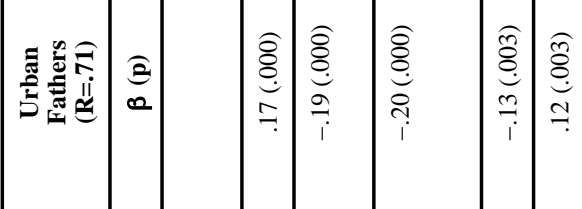

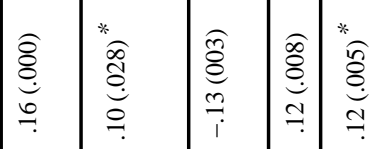

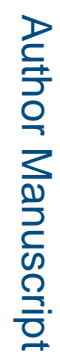

\begin{tabular}{|c|c|c|c|c|c|c|c|c|c|c|c|c|c|c|c|c|}
\hline 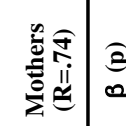 & 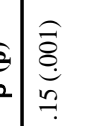 & & 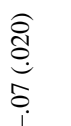 & 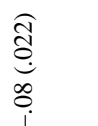 & & 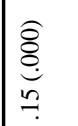 & & & $\begin{array}{l}\stackrel{0}{0} \\
\substack{0 \\
0}\end{array}$ & & & & 胥 & & & \\
\hline 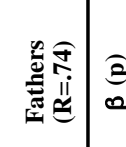 & 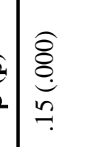 & 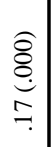 & 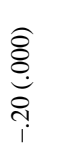 & $\begin{array}{c}\widehat{o} \\
\substack{n \\
i} \\
i\end{array}$ & $\begin{array}{l}\underset{\delta}{o} \\
\underset{.}{0} \\
\infty \\
i \\
i\end{array}$ & 产 & 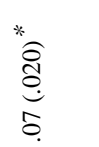 & 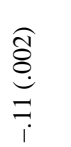 & 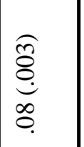 & 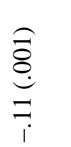 & 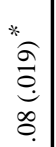 & 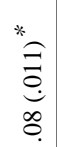 & 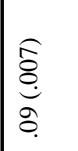 & & 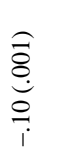 & \\
\hline 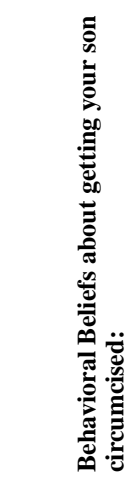 & 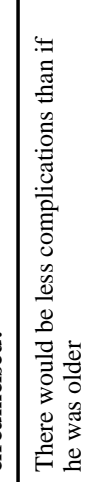 & 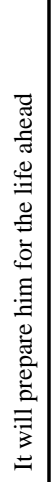 & 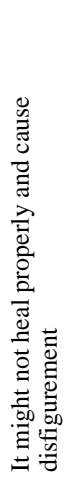 & 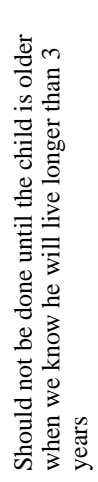 & 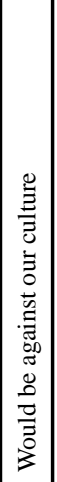 & 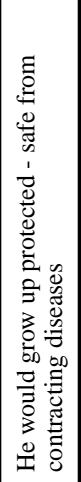 & 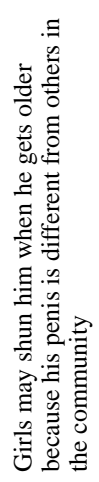 & 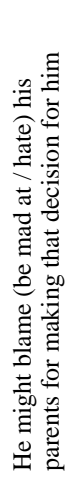 & 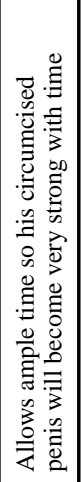 & 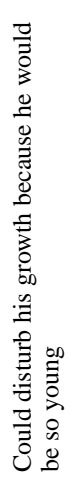 & 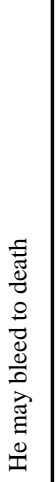 & 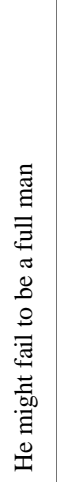 & 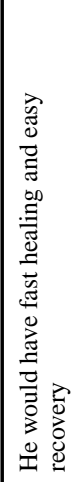 & 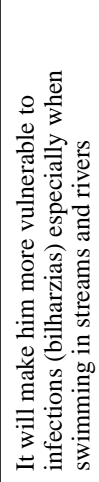 & 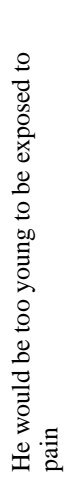 & 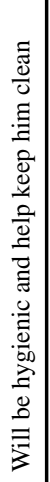 \\
\hline
\end{tabular}

AIDS Behav. Author manuscript; available in PMC 2019 February 01. 


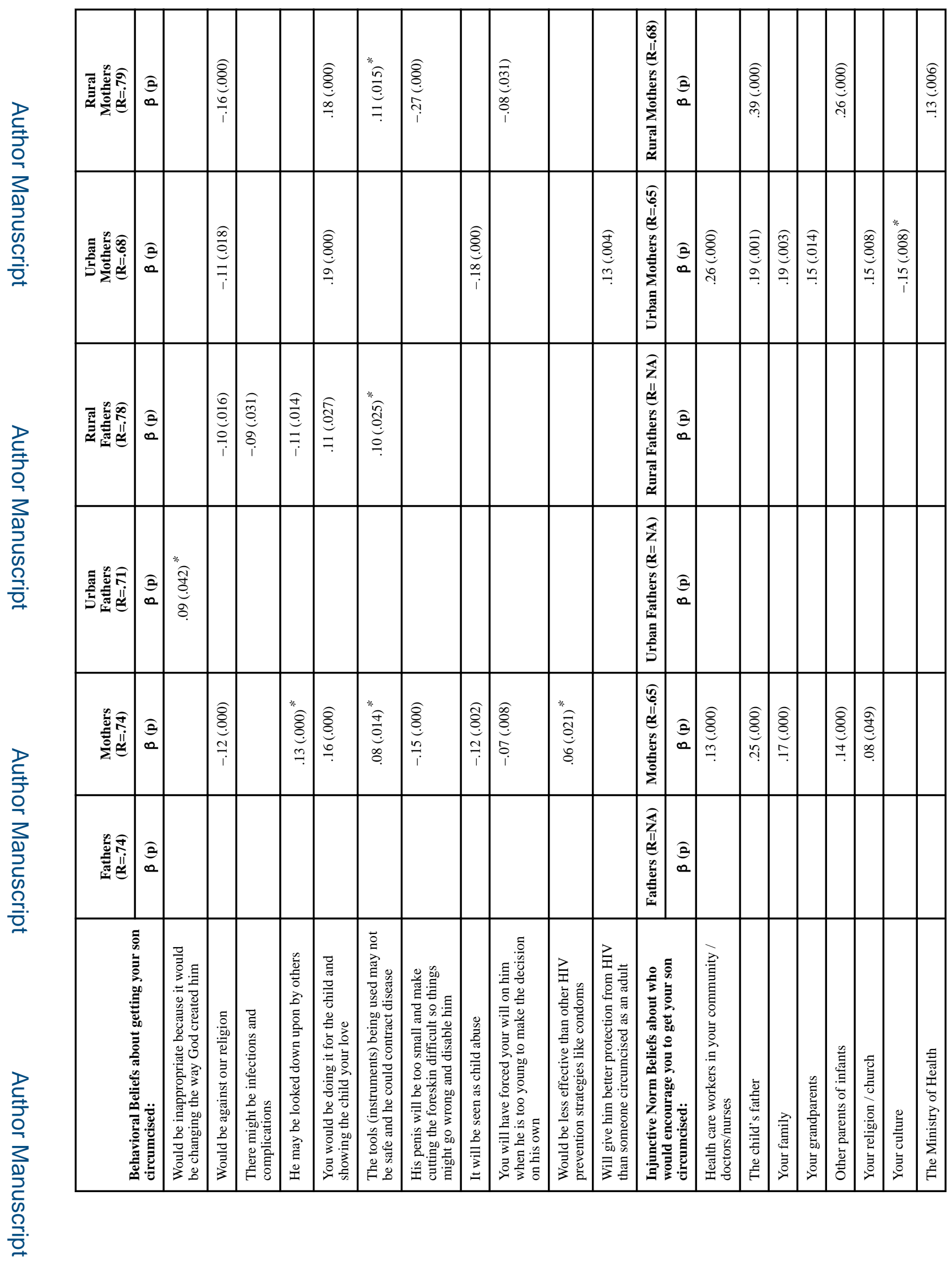

AIDS Behav. Author manuscript; available in PMC 2019 February 01. 


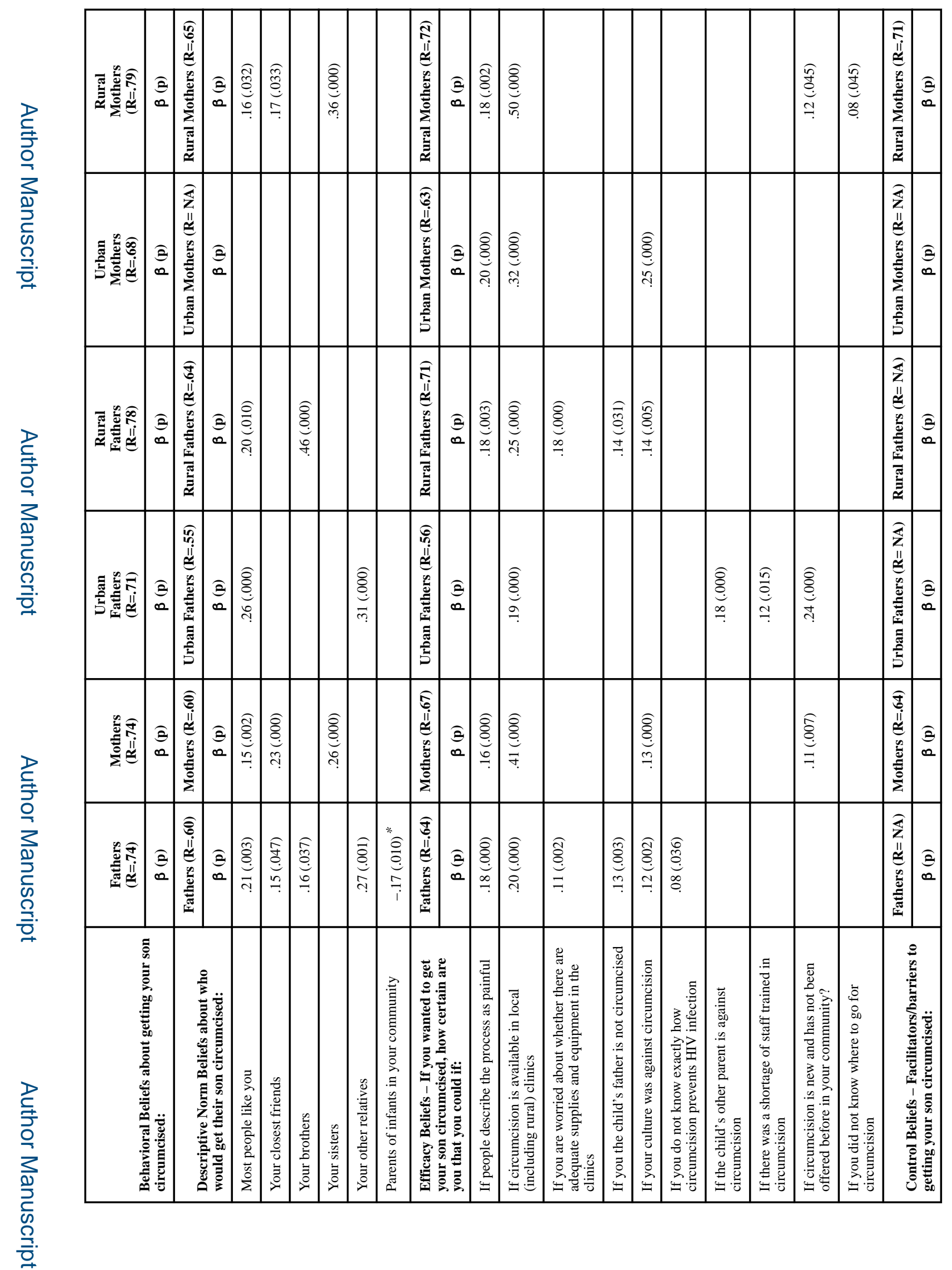

AIDS Behav. Author manuscript; available in PMC 2019 February 01. 


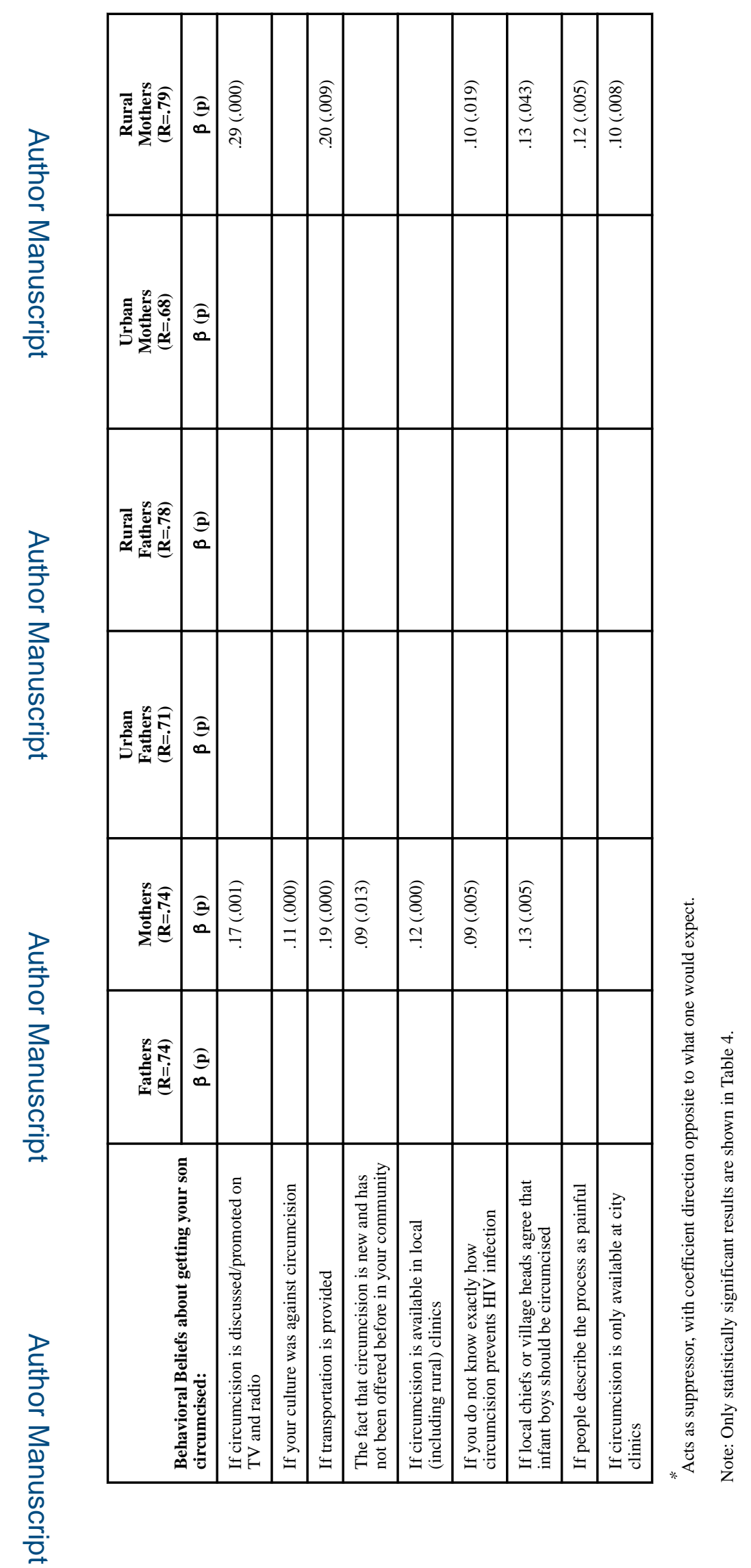

AIDS Behav. Author manuscript; available in PMC 2019 February 01. 


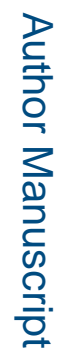

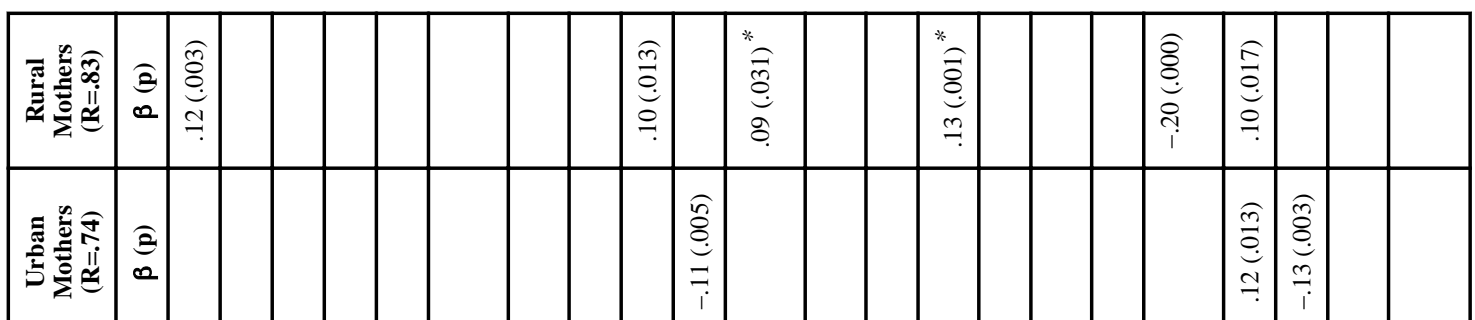

颃

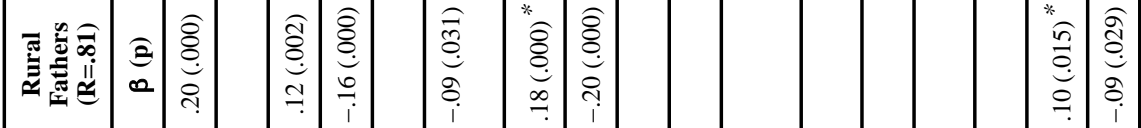

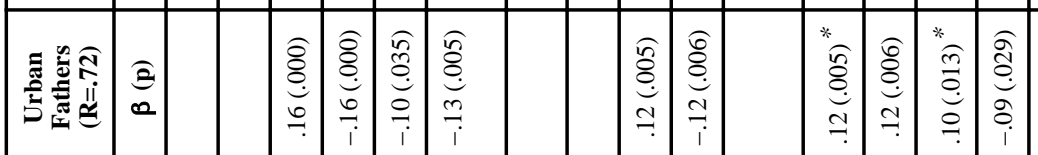

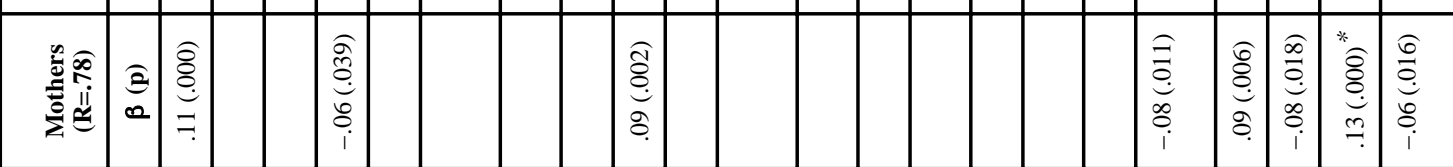

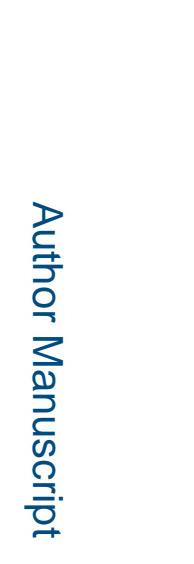

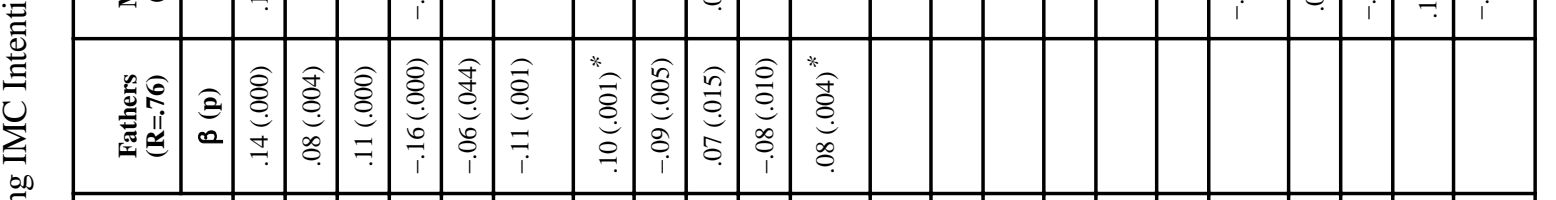

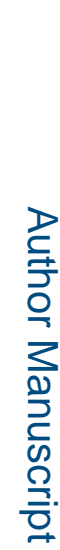
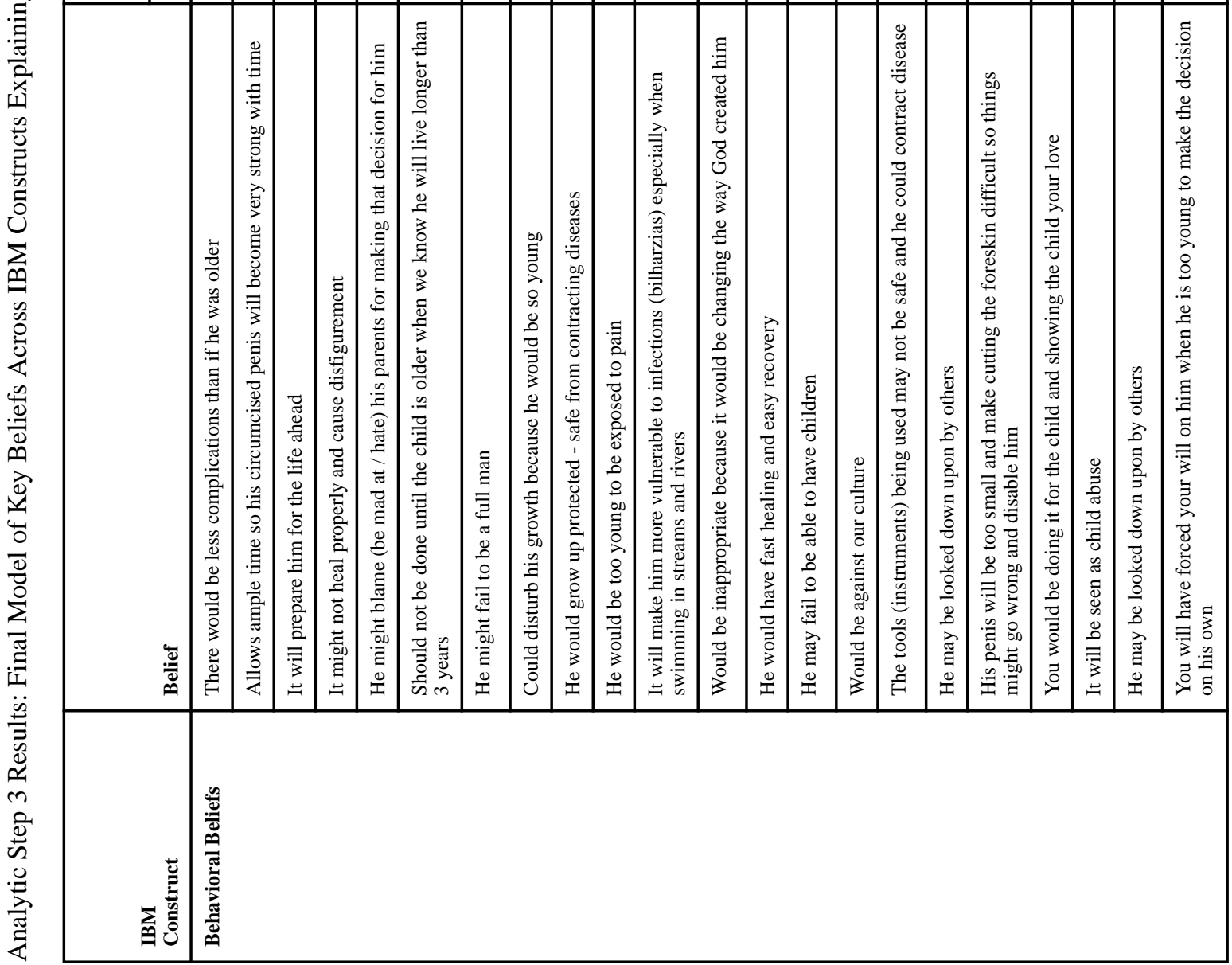

AIDS Behav. Author manuscript; available in PMC 2019 February 01. 


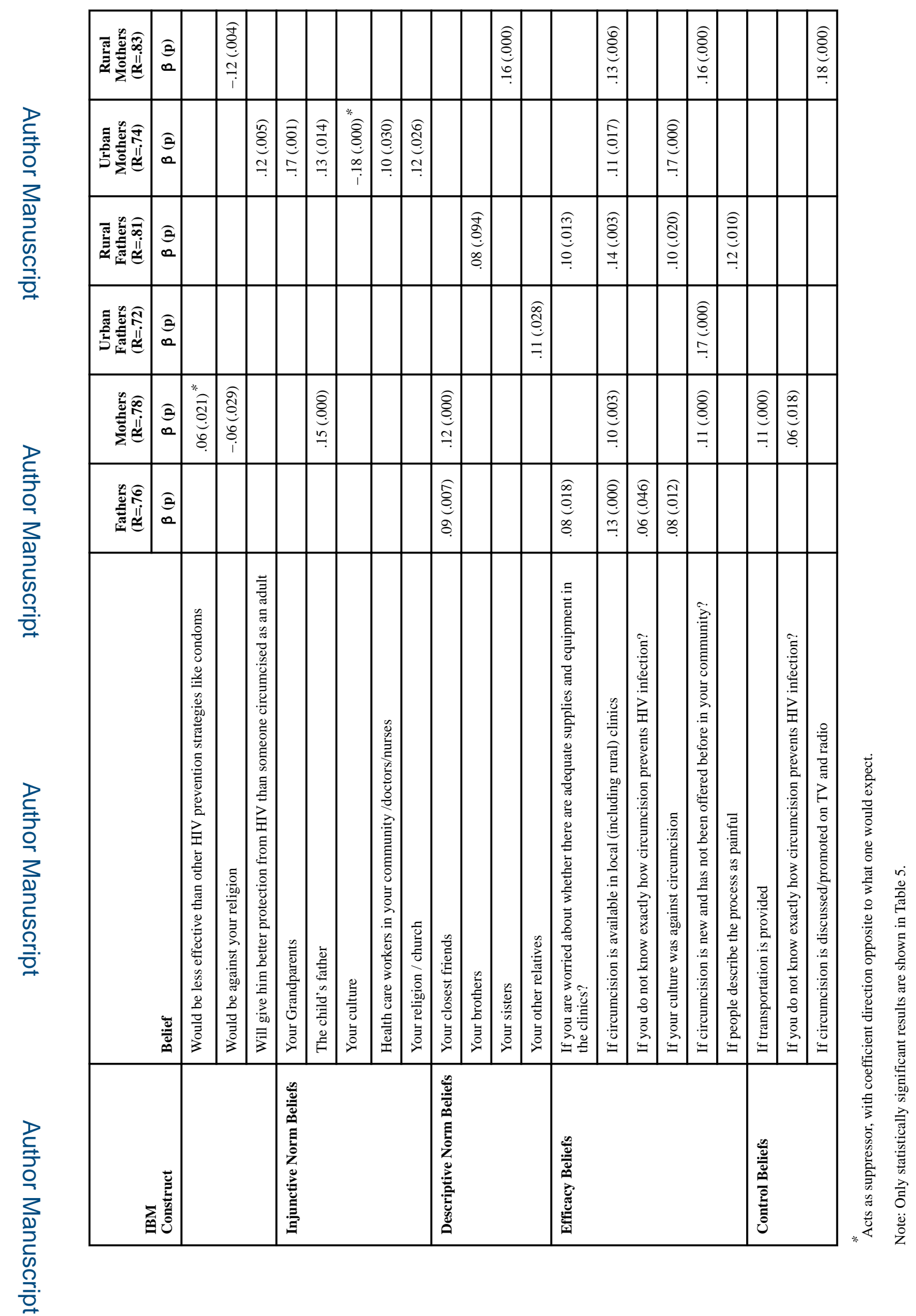

AIDS Behav. Author manuscript; available in PMC 2019 February 01. 